\title{
Evaluation of Human Safety in the DLR Robotic Motion Simulator using a Crash Test Dummy
}

\author{
Karan Sharma, Sami Haddadin, Sebastian Minning, Johann Heindl, Tobias Bellmann, Sven Parusel, Tim Rokahr \\ and Alin Albu-Schaeffer
}

\begin{abstract}
The DLR Robot Motion Simulator is a serial kinematics based platform that employs an industrial robot (as opposed to the conventional 'Hexapod') to impart motion cues to the attached simulator cell. This simulation platform is the culmination of ongoing research on motion simulation at the Robotics and Mechatronics Center, German Aerospace Center (DLR). Safety tests were undertaken to ascertain the effects of critical motions and subsequent emergency stop procedures on the prospective human passengers of the simulator cell. To this end, an Anthropomorphic Test Device (ATD) aka 'crash test dummy' was used as a human surrogate for these tests. Several severity indices were evaluated for the head-neck region, which was found to be more susceptible to injuries compared to the rest of the body. The results of this study are discussed in this paper.
\end{abstract}

\section{INTRODUCTION}

Motion simulation systems enjoy widespread applications in fields ranging from entertainment industry to defense research. In the domain of flight simulation, motion simulators have been in use since decades for pilot-training and research. The majority of these simulators employ variants of the six cylinder Stewart platform aka Hexapods to generate the necessary motion cues. This platform has a parallel kinematic configuration that allows motion in six degrees of freedom [1]. While these parallel kinematics based simulators can handle large loads e.g. complete cars [2] and hence provide for enhanced realism during simulations, they are expensive to deploy and offer a limited range of motion.

In recent years, KUKA robotics has introduced several heavy duty serial kinematics based industrial manipulators such as the KR-500, KR-1000 'Titan' etc. which have payloads of $500 \mathrm{~kg}$ and $1000 \mathrm{~kg}$, respectively. It was envisioned that, since the workspace of a KR-500 is larger and its also cheaper than a Stewart platform based simulator, it could be used as a motion simulation platform. The KUKARobocoaster ${ }^{1}$ was the first manifestation of this idea. It is a passive (i.e. preprogrammed) motion simulator that can sit up to two passengers and is primarily used as a amusement ride at several theme parks [3]. Since then, the partnership between DLR and KUKA has led to several iterations of the Robocoaster, e.g. KUKA RoboSim 4-D simulator [4]. It is a passive motion simulator that has been developed at DLR [5]. Besides the usual features offered by the Robocoaster,

*This work is supported by KUKA Roboter GmbH

The authors are with the Robotics and Mechatronics Center, German Aerospace Center (DLR), 82234 Oberpfaffenhofen-Wessling, Germany karan.sharma@dlr.de

${ }^{1}$ Copyright: KUKA Roboter GmbH

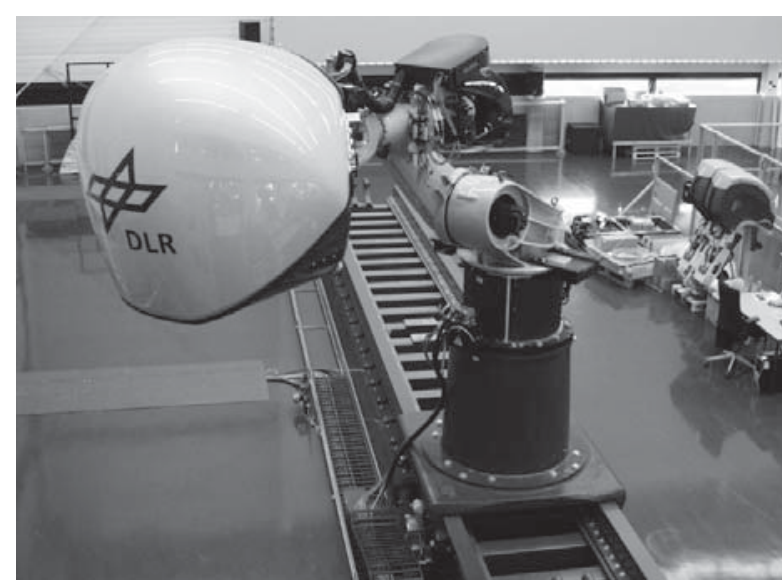

Fig. 1. DLR Robotic Motion Simulator (DLR-RMS)

this version includes a completely overhauled simulator cell that offers a better Audio/Video experience. This version has also been adapted for active (online) motion simulation and the underlying research has been addressed in [6], [7].

The latest iteration is known as the DLR Robotic Motion Simulator (DLR-RMS) [8]. The design and setup of this simulator was formulated based on experiences gained from earlier iterations of this simulation platform. Major modifications include a linear axis at the base of the manipulator, which extends the workspace of the simulator and a completely overhauled simulator cell design (Fig. 1 \& Fig. 2). This cell is inherently modular and allows usage of various instrument consoles as per the requirements of different simulation scenarios. This version has been primarily designed for active (passenger controlled) simulation and is capable of simulating land vehicles [7], airplanes [9] etc. Besides this, it also includes 3-D projection capabilities, several safety features, new seating setup etc.

The passenger seating setup of the RoboSim 4-D simulator was designed for passive simulation scenarios, so it featured top closing roller-coaster type seats. These seats are constricting and therefore not suitable for active simulation scenarios. Hence, the seating arrangement in the DLR-RMS was modified to make it more suitable for active simulation scenarios. The passenger can freely move his hands and has an unconstrained view of the instrument console.

Passenger safety is of paramount importance in every motion simulator. Initial attempts to determine the safety- 


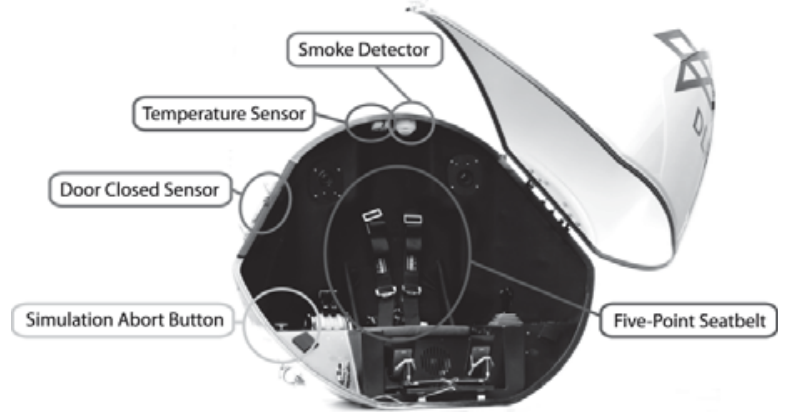

Fig. 2. Simulator Cell and some of its safety features

worthiness of serial kinematics based motion simulators were made using the RoboSim 4-D simulator [8]. Typical testing scenario involved driving the robot axes at high velocities and subsequently triggering an emergency stop (e-stop) to generate decelerations of the simulator cell. An inertial measurement unit (IMU), mounted near the usual head position inside the cell was used to measure the resulting decelerations. The peak value of e-stop deceleration during one of the tests was $3 \mathrm{~g}$ (including gravity). This is within the $5 \mathrm{~g}$ (harmless acceleration) limit for whiplash related injuries defined by a German jurisdiction [10]. Further investigation of the results revealed that these tests are not truly representative of the accelerations that would've been experienced by the passenger, as the sensor was mounted on the cell's enclosure. This way, the effects that would've been induced by the motion of head relative to the cervical spine and the torso, are not observed. Secondly, injury assessment criterion used in this investigation is not universally applicable to all types of high deceleration scenarios. It is supposed to serve as rough assessment criterion for disorders resulting from rear impacts (explained in section II).

Based on the results of these initial tests, several software based safety measures (together referred to as 'watchdog application') were developed and integrated into the control architecture to provide an extra layer of security [11]. This watchdog application continuously observes the state and motion profile of the manipulator to predict and prevent an impending high deceleration scenario (such as a software triggered e-stop). To analyze the accelerations experienced in the head-neck region, an anthropomorphic test device (ATD) popularly known as 'crash test dummy' [12] was used for the subsequent safety evaluation mentioned in [11]. This work was an important step in the safety analysis of the DLRRMS, but was limited by type and number of sensor data (only accelerations) available for evaluation.

In this paper, a more detailed and thorough analysis of passenger safety in the simulator is presented. The usage of multiple types of sensors in the ATD e.g. acceleration, force/torque as opposed to only acceleration sensors in the earlier analyses, allows for a broad range of data from which values for several different injury indices are evaluated. Also, other characteristics of the experiments e.g. e-stop type, motion profile etc. are widely varied to facilitate for a more comprehensive analysis from a diversified set of data. For the tests presented in the following sections (refer Sec. V), the watchdog application is not used. This application is currently under further development and is not very robust. By not employing this application for these experiments, one can account for scenarios where this application could fail to detect a critical situation. This allows for a more rigorous evaluation of the general safety setup.

The field of serial kinematics based motion simulation is currently in its initial stage. In the past years, the range of simulation scenarios has expanded from passive to active and new simulation scenarios are being actively pursued [13]. However, in the knowledge of the authors, the issue of passenger safety has not yet been thoroughly addressed. This work aims to bridge this gap by undertaking a comprehensive safety analysis for such a simulator. Through this evaluation, we hope to identify and subsequently address any critical scenarios that affect human safety. Thereby also addressing issues that could impede the further development of this field.

In the following section, the critical scenarios that a passenger could face during a simulation are introduced. This is followed by an introduction to different injuries and their evaluation indices. Then the experimental setup is described and finally a discussion of the results is presented.

\section{CRITICAL SCENARIOS}

As established earlier, the DLR-RMS employs a manipulator to produce the motion cues during simulation. The safety setup of these manipulators is designed for industrial settings, where the aim of this setup is to immediately stop the manipulator and prevent its axes from colliding with each other. To achieve this, they are equipped with brakes and several e-stop procedures (refer Sec. IV). But, what happens when a human is seated at the Tool Center Point (TCP) of such a manipulator and one of these e-stops is executed? To analyze these scenarios, first the classification of accelerations that are critical to humans is presented as follows:

- Impact accelerations: acceleration pulses of up to 200ms are classified as impacts e.g. high decelerations due to car crashes, resulting accelerations when slapping someones back etc.

- Sustained accelerations: accelerations lasting longer than 200ms are classified as sustained accelerations e.g. in roller-coasters, fighter planes etc. These accelerations (in a range of few $\mathrm{g}$ ) can lead to G-Induced Loss of Consciousness (G-LOC) e.g. an acceleration with an onset rate of $0.5 \mathrm{~g} / \mathrm{s}$ in the $+\mathrm{Gz}$ direction (upwards acceleration, that pushes the body back into its seat) can lead to G-LOC within 6s [14].

The manipulator used for the simulator is constrained by its joint limits and hence, is not capable of producing sustained accelerations in one direction that could lead to G-LOC. It can however produce high accelerations during impacts, which can be further categorized into: 


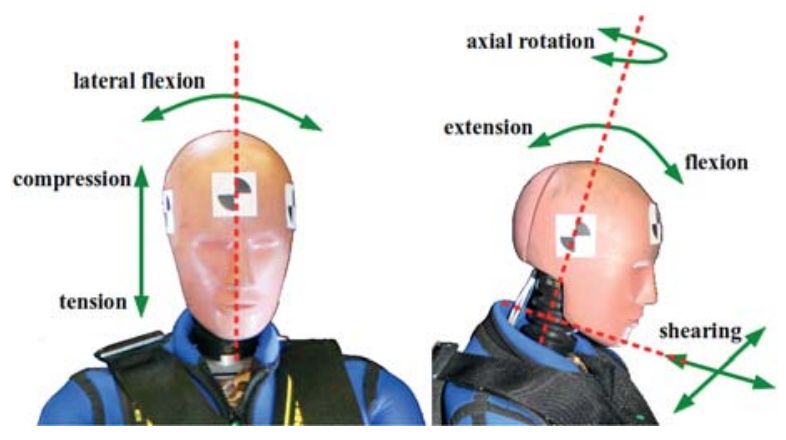

Fig. 3. Taxonomy of the 'Head-Neck' motions

- Direct impacts: result in high accelerations experienced by the passenger (i.e. the ATD in this scenario) due to a collision with a surface [15] e.g. head of an auto-mobile driver colliding with the steering wheel during a crash.

- Inertial loading (aka indirect impact): 'loading' produced in head-neck complex due to sudden changes in the motion of the torso is an example of an inertial loading scenario. The motion of the head relative to the torso leads to straining of the neck and can lead to a multitude of injuries in the head-neck region [16].

While direct impacts are possible in the DLR-RMS, such collisions can only result from human error e.g. seat-belt not used, console not mounted correctly etc. In these scenarios the passenger is susceptible to injuries, but these injuries shouldn't be classified as resulting from the simulator. The safety setup of the DLR-RMS is designed to prevent all foreseeable direct impact situations [8].

Whiplash associated disorders (WADs) is a term used for a group of injuries that are essentially caused by inertial loading of the head-neck complex. Traditionally, the term WADs was used to refer to injuries that are experienced by the passengers of a vehicle during rear impacts i.e. when their vehicle is impacted from the back by another vehicle/object [17]. Today, the term WADs is extended to all disorders resulting from different types of inertial loading i.e. front, rear and side-ward impacts [18]. During initial experimentation, it became evident that the human passenger inside the simulator cell is most susceptible to inertial loading, when an e-stop is triggered [8] [11]. In the following subsection, an overview of inertial loading scenarios for the head-neck complex is presented.

\section{A. Response of Head-Neck complex under Inertial loading}

The decelerations applied during an impact determine the direction of inertial loading. These decelerations result in a displacement of the head relative to the torso, which in turn leads to bending moments and forces at the head-neck and neck-torso junctions. A front impact [19] (i.e. a headon collision of a vehicle with another object/vehicle) and a rear impact typically results in flexion/extension motion of the head-neck complex (Fig. 3). Similarly, side impacts primarily result in lateral flexion movements but also induce axial rotations. This is due to the complex dynamics of the head-neck system which exhibits different characteristics under different loading scenarios [20]. All impacts (frontal, rear and side) lead to shearing forces that vary as per the nature of the impacts.

In the automotive industry, several indices are used to determine the probability of injuries resulting from inertial loading during car crashes. The criteria deemed suitable for analysis of inertial loading scenarios in the DLR-RMS are presented in the next section.

\section{INJURY INDICES}

The indices used for injury analysis can be classified based on the nature of inertial loading. Further, different criterions are used for injury analysis of the head and neck regions. The indices used for safety analysis in DLR-RMS are as follows:

\section{A. Injury indices for the Head}

Head Injury Criterion (HIC) is used to analyze the effects of inertial loading on the head [15]. This index can be used for situations involving both inertial loading and direct impacts (collisions). Another advantage of this criterion is that it can be used to analyze front, rear and side impacts. This makes it one of the most widely used index for evaluation of head injury. HIC is calculated in two different ways. The time window around the peak acceleration values is varied in order to maximize the HIC value [21]. This window can either be $15 \mathrm{~ms}$ or $36 \mathrm{~ms}$ and the $\mathrm{HIC}$ values pertaining to these are referred to as $H I C_{15}$ and $H I C_{36}$, respectively [22]. $H I C_{15}$ has been determined to be more restrictive than $H_{I} C_{36}$ [15] and therefore the latter is used for injury analysis in our experiments. The following equation is used to calculate $H I C_{36}$ :

$$
\begin{aligned}
& H I C_{36}=\max _{\triangle t}\left\{\triangle t\left(\frac{1}{\triangle t} \int_{t_{1}}^{t_{2}}\left\|\ddot{x}_{H}\right\| \mathrm{d} t\right)^{\left(\frac{5}{2}\right)}\right\} \\
& \text { with: } \triangle t=t_{2}-t_{1} \leq \triangle t_{\max }=36 \mathrm{~ms}
\end{aligned}
$$

$\left\|\ddot{x}_{H}\right\|$ is the norm of acceleration of the human head, measured in $\mathrm{g}=9.81 \mathrm{~m} / \mathrm{s}^{2}$.

According to EuroNCAP protocol that is used for injury assessment in automotive crashes [23], the critical limit for $H I C_{36}$ that can result in 'serious injury' is 1000 [15].

\section{B. Injury indices for the Neck system}

Depending upon the inertial loading scenario (refer Sec. II-A), different indices are used to ascertain the severity of loading on the neck region. They can be classified into the following types:

1) Front impacts: during these impacts the head-neck complex undergoes flexion and during rebound may also experience extension. To examine the consequences of this impact, the EuroNCAP criterion for neck injury analysis in front impact scenarios [19] is used. The corresponding critical limits are defined with respect to the positive cumulative exceedance time. The values mentioned in the Table I 
are linearly interpolated to define a critical limit that varies with time [15]. For extension, only the initial measurement(at $0 \mathrm{~ms}$ ) is relevant.

TABLE I

NIC LIMITS FOR FRONT IMPACTS

\begin{tabular}{|l||c|c|r|}
\hline \multicolumn{1}{|c||}{ Type } & \multicolumn{3}{c|}{ Critical value (lower/upper limit) } \\
\hline \hline & @0ms & @ 25-35ms & @ 45ms \\
\hline Shear & $1.9 / 3.1 \mathrm{kN}$ & $1.2 / 1.5 \mathrm{kN}$ & $1.1 / 1.1 \mathrm{kN}$ \\
\hline Tension & $2.7 / 3.3 \mathrm{kN}$ & $2.3 / 2.9 \mathrm{kN}$ & $1.1 / 1.1 \mathrm{kN}$ \\
\hline Extension & \multicolumn{3}{|c|}{$42 / 57 \mathrm{Nm}$ (only @0ms) } \\
\hline
\end{tabular}

2) Rear impacts: like front impacts, the head-neck complex in this case undergoes flexion and extension. But the order of onset of these displacements is reversed i.e. the complex first undergoes extension and in certain cases flexion (e.g. as a result of rebound from the head-rest) is also observed. Several injury assessment criteria are used to evaluate loading of the neck region resulting from rear impacts. In [16] a comparative study of several of these criterions is presented. The Neck Injury Criterion (NIC) proposed by Bostroem [24] is used for the following experiments. The formula used for calculating NIC is as follows:

$$
N I C=a_{\text {relative }} * 0.2+v_{\text {relative }}^{2}
$$

where $a_{\text {relative }}=a_{x}^{T 1}-a_{x}^{\text {Head }}$ and $v_{\text {relative }}=\int a_{\text {relative }}$. Here, $a_{x}^{T 1}$ is the acceleration of first chest vertebra in x-direction and $-a_{x}^{\text {Head }}$ is the acceleration at the center of gravity of the head in $\mathrm{x}$-direction. The tolerance level of NIC(rear impact) is defined as $15 \mathrm{~m}^{2} / \mathrm{s}^{2}$.

3) Side impacts: most automotive side impact assessments don't evaluate loading on the neck resulting from lateral flexion [20]. The criteria defined by EuroNCAP for side impacts only addresses loading scenarios pertaining to the following body regions: head, ribs, abdomen, pelvis and the pubic symphysis [25]. Initial attempts to ascertain the response of the human head-neck complex under lateral bending are presented in [20]. A couple of Post-mortem Human Subjects (PMHS) and a dummy were subjected to lateral flexion movements. The peak magnitudes of various forces and moments applied to head-neck junction during these tests are presented in Table II. The values listed in this table don't represent critical values and these loading conditions didn't result in any fractures/injuries on the PMHS. In a later publication [26], a initial toleration limit of $75 \mathrm{Nm}$ was identified for lateral flexion loading. For these experiments this limit is used.

\section{EXPERIMENTAL SETUP}

The instrumentation and the placement of the dummy is shown in Fig. 4. The dummy used for these experiments is a EuroSID (European Side Impact Dummy) equipped with several acceleration and force-torque (load cells) sensors, as shown in Fig. 5. A sampling rate of $20 \mathrm{kHz}$ is used for data acquisition and the data is filtered as per the guidelines mentioned in [23]. Two video cameras, one in slow motion
TABLE II

PEAK MAGNITUDES FOR LOADING

\begin{tabular}{|c||c|c|c|}
\hline Variable & PMHS1 & PMHS2 & dummy \\
\hline \hline Fx $(\mathrm{N})$ & -511 & -458 & -233 \\
\hline Fy $(\mathrm{N})$ & 1297 & 639 & 732 \\
\hline Fz $(\mathrm{N})$ & 1193 & 1498 & 891 \\
\hline Mx $(\mathrm{Nm})$ & 81.8 & 67.1 & 49.0 \\
\hline My $(\mathrm{Nm})$ & -27.7 & -25.6 & -7.7 \\
\hline Mz $(\mathrm{Nm})$ & 26.8 & 26 & 26.4 \\
\hline
\end{tabular}

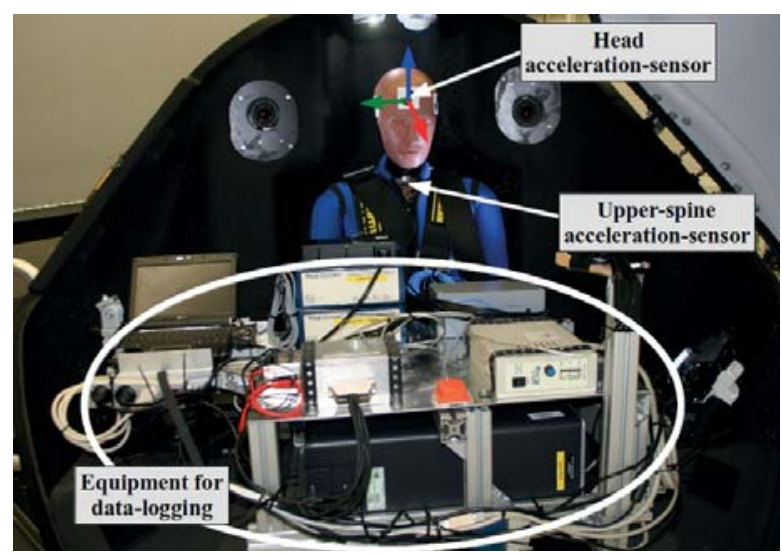

Fig. 4. Dummy and data acquisition equipment in the cell

mode and the other in normal mode, were installed inside the cell to record the motions of the dummy. Another camera was placed at a convenient location afar to record the motions from outside the cell.

During normal simulation scenarios, motion cues are generated based passenger input. The cues received from the user input device (e.g. joystick) are first optimized to the workspace of the simulator [9] and then transmitted to the manipulator controller using KUKA-Robot Sensor Interface (RSI) [27]. This software framework facilitates the execution of externally generated trajectories by the simulator.

For these experiments (and the initial tests mentioned in section I), the motion profiles are generated on an external computer and 'commanded' to the robot controller over an Ethernet connection. During the course of these motions an e-stop is triggered (either via software or through an hardware button), which brings the manipulator to a halt. The resulting decelerations, forces and torques generated during the stopping motion are recorded by the dummy. The motion profiles for the following experiments were developed to instigate the critical scenarios mentioned in section II-A.

The underlying manipulator (KR-500) and it's control architecture (KRC) offers 3 types of e-stops: STOP 0, STOP 1 and STOP 2 [28]. These stops are also available within the RSI framework [27]. A short description of these e-stops and their nomenclature within RSI is as follows:

- Normal Stop: for the manipulator, this stop is referred to as 'Ramp-down braking (STOP 2)'. Both the joint drives and brakes remain open; the joints are stopped using a normal ramp for deceleration [28]. 


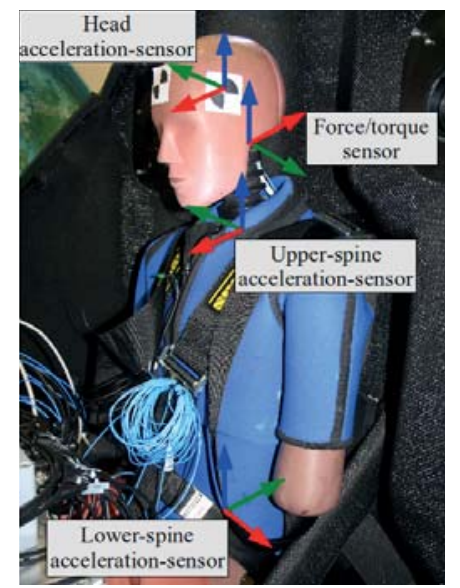

Fig. 5. Location of the various sensors within the dummy. In the co-ordinate system red: $\mathrm{x}$-axis, green: $\mathrm{y}$-axis and blue: $\mathrm{z}$-axis

- Velocity Stop: the equivalent standard stop for this type of braking is called 'Path-maintaining braking (STOP $0)$ '. The joints drives are switched off and brakes are immediately applied.

- Fast Stop: is a type of 'path maintaining braking (STOP 1)' procedure. During the first second the controller brakes the robot using a steeper stop ramp [28]. After this $1 \mathrm{~s}$, the drives are switched off and the brakes are applied.

\section{EXPERIMENTS}

The experiments can be classified into two types. These are: Rare and Plausible scenarios. Both feature motion trajectories that are characterized by an initial acceleration phase followed by the onset of sudden deceleration (using an e-stop). What differentiates them however, is the way in which the e-stop is triggered and the probability of their occurrence during a simulation.

The rare scenarios presented here have been especially designed for the following experiments. The probability of their occurrence during normal simulation operation is very low because these kinds of motion trajectories are usually only possible if they have been explicitly programmed by the programmer/user. For the rare scenarios presented in the sub-section $\mathrm{V}$-A, the e-stops are pre-programmed and are usually triggered at the end of the acceleration phase. These experiments were undertaken three times and for each of these 3 iterations, a different type of e-stop was used. Therefore, the resulting effect (on the dummy) for each of these braking procedures was evaluated.

Plausible scenarios on the other hand, can occur whenever the safety setup of the robot detects an anomaly and reacts by inducing an e-stop. Due to space constraints, only a condensed explanation of the these experiments is presented. The various experiments are listed as follows:

\section{A. Rare Scenarios}

- Experiments $1 \& 2$ : for the first experiment, joints $q_{2}, q_{3}$ and $q_{5}$ are initially driven to their negative joint limits

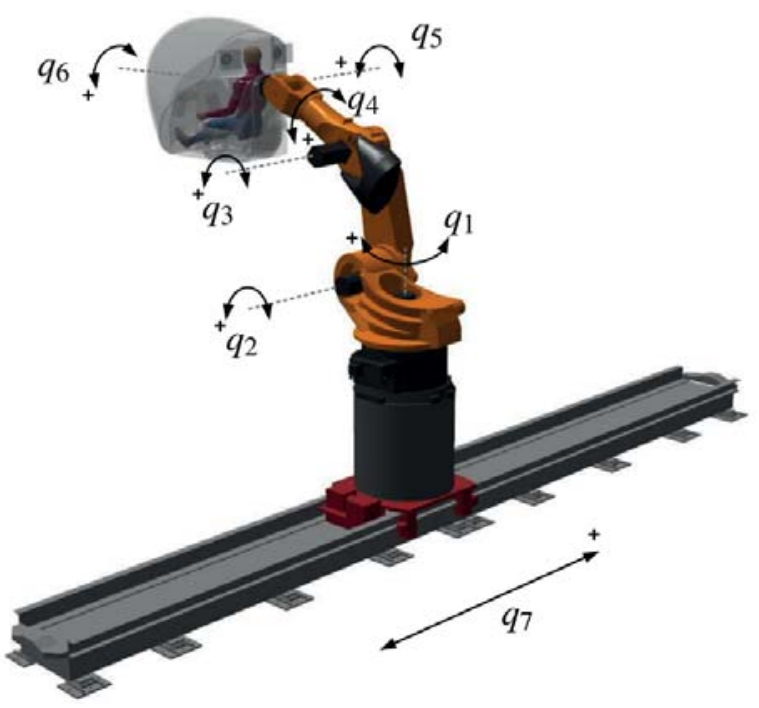

Fig. 6. Schematic showing joint locations and turning directions of the manipulator

(refer Fig. 6). This results in the high up position as shown in Fig. 7. From this position, these joints are programmed to synchronously move in the direction of their positive joint limits. During the course of this motion, as soon as the joints achieve their maximum velocities, an e-stop is triggered. Experiment 2 is the same as experiment 1 , but executes the motion in the opposite direction. The aim of these experiments is to induce flexion and extension motion on the human headneck complex i.e. conditions similar to those observed during front and rear impacts (refer Sec. II-A).

- Experiments $3 \& 4$ : involve moving the joints $q_{1}$ and $q_{7}$ from one end of their joint limits to the other end (Fig. 8). In Exp. 3 the joints are driven from the positive(+ve) to the negative(-ve) end and in Exp. 4, the vice-versa. For these experiments, the e-stop is triggered when the joints reach a specific preprogrammed position, which in this case was the middle point of the joint extremes. The aim of this set of experiments is to induce lateral flexion on the head-neck complex i.e. conditions similar to those observed in side impacts (refer Sec. II-A).

- Experiments 5 \& 6: are similar to experiments 3 \& 4, but undertaken by only moving joint $q_{1}$.

- Experiments 7 \& 8: are similar to experiments 3 \& 4 . But in these experiments, the e-stop is executed when the joints synchronously reach their maximum joint velocities.

- Experiments $9 \&$ 10: similar to experiments $5 \& 6$. The stop is triggered when the joints reach their maximum velocities (as in experiments $1 \& 2$ ).

- Experiments 11 \& 13: for these experiments, the joints $q_{4}$ and $q_{6}$ are moved from their +ve to -ve joint limits and vice-versa, respectively. The e-stop is triggered when the joints achieve a pre-determined position. The aim of these tests is to primarily induce lateral flexion 
scenarios. But as it was explained in Sec. II-A, due to the complex nature of the head-neck region, motions can also be observed in other loading directions.

- Experiments 12 \& 14: are similar to experiments 11 \& 13. For these experiments, the e-stop is triggered when the joints synchronously reach their maximum velocities.

As already mentioned in section IV, the manipulator is seldom driven at maximum joint velocities. During an online simulation, the software setup aims to keep the manipulator in a configuration that facilitates for better execution of the commanded motion cues [9] thereby restricting the kind of motions generated for experiments 1-14. So, we reiterate that rare scenarios are worst case situations that have been used here for stress testing.

\section{B. Plausible scenarios}

- Experiments 15-16-17: For these experiments, point-topoint (PTP) motions at different velocities and accelerations were executed on the manipulator [29]. During the course of these motions, e-stops were triggered by:

- Pressing the hardware emergency stop located in the simulator control room [8].

- Interrupting the Ethernet communication by removing the cable from the external computer; and

- Opening the gates of the safety cell that surrounds the motion simulator. This was undertaken to simulate an unauthorized entry into the workspace of the simulator.

- Experiment 18: before the start of a motion simulation, the manipulator moves from its home position to the 'simulation start' position. This motion has to be executed by a simulation supervisor using the robot control pendant [29]. For the duration of this motion, a safetyswitch (also called an 'enabling button') needs to be continuously pressed. In this test, the safety switch was released during this initial motion (known as 'BCO run' [29]), leading to an e-stop. This experiments aims to analyze the resulting loading on the dummy during such stops.

As can be observed, the probability that these situations occur during a simulation scenario is higher than for the situations mentioned under rare scenarios.

TABLE III

INDICES USED FOR THE EXPERIMENTS

\begin{tabular}{|c||c|c|c|c|}
\hline Experiment & HIC $_{36}$ & NIC & SIBM & NCAP front \\
\hline \hline 1 & $\mathrm{x}$ & - & - & $\mathrm{x}$ \\
\hline 2 & $\mathrm{x}$ & $\mathrm{x}$ & - & - \\
\hline $3-18$ & $\mathrm{x}$ & - & $\mathrm{x}$ & - \\
\hline
\end{tabular}

\section{EXPERIMENTAL RESULTS}

Table III lists the different indices that have been used for evaluation of inertial loading on the head-neck complex. For head injury analysis, $H I C_{36}$ is used for all the

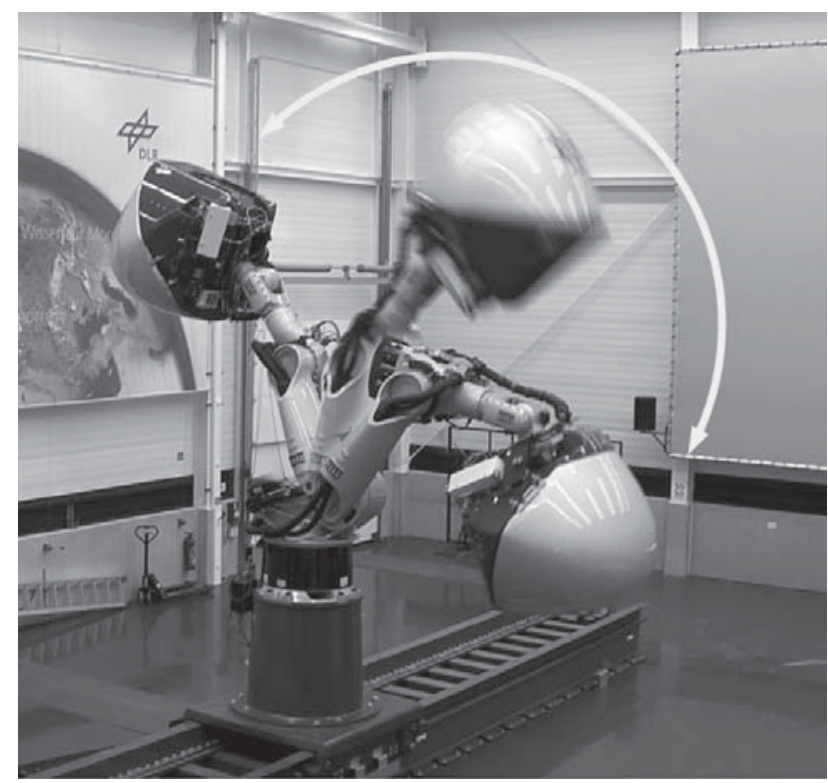

Fig. 7. Movement of the simulator during experiments $1 \& 2$

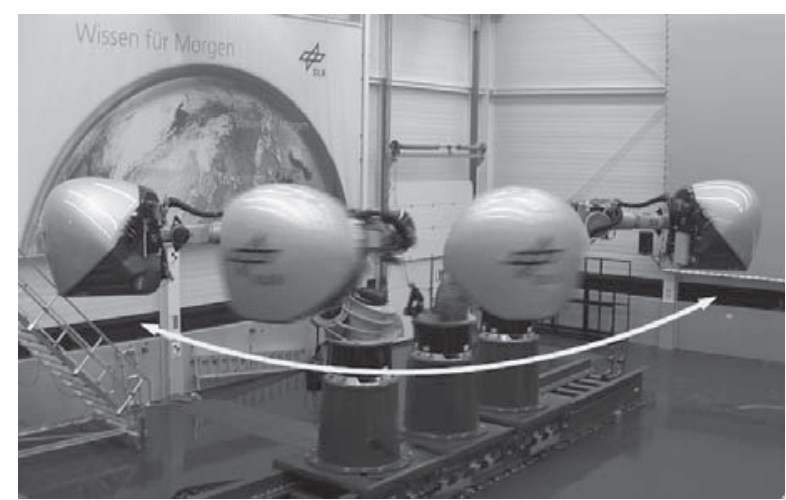

Fig. 8. Movement of the simulator during experiments $3,4,7 \& 8$

experiments. For neck injury/loading analysis, experiment 1 employs EuroNCAP's NIC criterion for frontal loading [19] and experiment 2 uses the NIC criterion from Boestrom [24]. Experiments 3-18 employ an 'initial' injury assessment parameter (abbr. as SIBM in the table) defined in [20] [26] for assessment of loading on the neck region.

\section{A. Results for the Head}

The critical value defined by the EuroNCAP for $H I C_{36}$ is 1000 [23]. The maximum $H_{I} C_{36}$ value evaluated for these experiments was 3.35 for experiment 15 and 3.15 for experiment 17 (ref Table IV). The values for the other experiments were far lower. The peak acceleration values during these tests were $7.37 \mathrm{~g}$ and $7.02 \mathrm{~g}$. A life threatening $H I C_{36}$ value of 1000 is achieved, when the peak acceleration is around $60 \mathrm{~g}[21]$.

\section{B. Results for the Neck}

Experiment 1 was developed to introduce flexion inducing motion during a e-stop. For this experiment, the resulting 
bending moment generated about the y-axis $\left(M_{y}\right)$ (refer Fig. 5) was evaluated to be $16.49 \mathrm{Nm} @ 0 \mathrm{~ms}$ (refer Table IV). This is distinctly below the limit of 42/57Nm@0ms defined by EuroNCAP [25]. For experiment 2, the resulting loading pattern is similar to the ones exhibited during rear-impacts. The $N I C$-whiplash criterion was evaluated to be $0.16 \mathrm{~m}^{2} / \mathrm{s}^{2}$ (refer Table IV). This value is substantially under the $15 \mathrm{~m}^{2} / \mathrm{s}^{2}$ critical limit.

Experiments 3 to 18 are similar to the side-impact scenarios that induce lateral flexion on the dummy's head-neck complex. Currently, there exists no well documented and widely used criterion for analyzing the loading on the neck region [20]. The index used here for injury evaluation is based on the initial findings in [26], which states a critical limit of $75 \mathrm{Nm}$ for bending moment $M_{x}$ about the x-axis (refer Fig. 5). For these experiments, the evaluated values were below this critical limit (refer Table V). Further investigation of data revealed an unusual bending moment $M_{z}$ (refer Fig. 9) about the z-axis for experiment 3 (marked with a * sign in Table V). This anomaly was attributed to a collision between the head and the side wall of the simulator cell, that resulted in an axial rotation.

TABLE IV

RESULTS FOR THE EXPERIMENTS 1 AND 2

\begin{tabular}{|c||c|c|c|}
\hline Experiment & HIC $_{36}$ & NIC & NCAP front \\
\hline \hline 1 & 2.34 & - & 16.49 Nm @ 0ms \\
\hline 2 & 0.91 & 0.16 & - \\
\hline
\end{tabular}

TABLE V

RESULTS FOR EXPERIMENTS 3-18

\begin{tabular}{|c||c|c|c|c|}
\hline Experiment & HIC $_{36}$ & Mx $(\mathbf{N m})$ & $\mathbf{M y}(\mathbf{N m})$ & $\mathbf{M z}(\mathbf{N m})$ \\
\hline \hline $3^{*}$ & 0.48 & 2.61 & 3.87 & 15.33 \\
\hline 4 & 0.46 & -4.75 & -2.17 & -1.96 \\
\hline 5 & 0.63 & 4.96 & 2.19 & 2.47 \\
\hline 6 & 0.57 & -4.59 & 1.50 & -2.58 \\
\hline 7 & 0.39 & -4.31 & 2.70 & 2.05 \\
\hline 8 & 0.84 & 4.59 & 2.53 & -2.18 \\
\hline 9 & 0.72 & 4.27 & 2.16 & 2.96 \\
\hline 10 & 0.63 & -3.88 & 1.24 & -1.61 \\
\hline 11 & 0.18 & 2.01 & -1.90 & -0.74 \\
\hline 12 & 0.38 & 4.67 & -2.05 & 1.75 \\
\hline 13 & 0.19 & 2.03 & -1.77 & 0.88 \\
\hline 14 & 0.41 & -4.50 & -1.80 & -1.63 \\
\hline 15 & 3.35 & -6.11 & 3.15 & 10.60 \\
\hline 16 & 1.18 & 6.49 & 9.45 & 6.95 \\
\hline 17 & 3.15 & 6.37 & -1.38 & 4.03 \\
\hline 18 & 0.06 & 2.74 & 1.96 & 1.24 \\
\hline
\end{tabular}

\section{CONCLUSION \& OUTLOOK}

Based on the $\mathrm{HIC}_{36}$ results listed in Tables IV and V, it can be stated that the probability of inflicting a life threatening head injury to the passenger is very low. In case of a mechanical failure, that leads to a situation where the robot crashes against itself or drives into the hardware limits; the values for $H I C_{36}$ would be much higher than

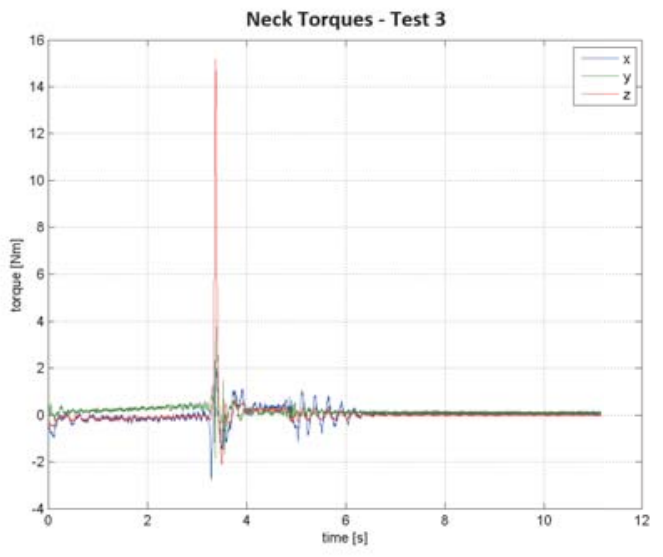

Fig. 9. Plot of the resulting torques in experiment 3

those evaluated in our results. Nevertheless, they would still be in a non-critical range as the motion simulator features hardware stops that absorb the energy of these impacts. As already mentioned in section II, injuries resulting from a direct impact with the interiors (e.g. the console) can't be attributed to the simulator as these injuries would be a direct result of hardware malfunction and/or human error. They are not explicitly caused due to the stopping procedures used by the simulator. Such direct impact scenarios should be avoided at all costs.

Upon comparing the results for the neck region (Tables IV and $\mathrm{V}$ ) with the injury assessment indices, it becomes evident that the probability of inflicting damage to the neck region is very low. The value of bending moment $M_{z}$ about the z-axis for experiment 3 (refer Table $\mathrm{V}$ ) is unexpectedly higher than the values for other experiments. The reason for the same was discussed in Sec.VI-B. Although this value is higher than expected, it is still non-critical (refer Sec. III-B and Table II). One reason for this is that walls of the simulator cell are coated with thick upholstery, that makes them very compliant during a collision. It should however be noted, that such scenarios where an inertial loading situation is transformed into a direct impact should be prevented. For the current scenario, it can be prevented by installing a better seat that fits the passenger properly and stops undesired motions of his/her torso. This issue will be addressed in the near future.

For experiments $15-17$, the values of $H_{I} C_{36}$ and bending moments are on an average higher than those for experiments 3-18. This unexpected result is attributed to the non-linear relation between joint velocity and braking distance. For high joint velocities the braking distance is larger than those for low joint velocities. When an e-stop is triggered during a motion being executed at low velocities, the braking distance is shorter and this results in higher decelerations at the joints. These comparatively higher decelerations lead to higher values of $H I C_{36}$ and bending moments for experiments 15-17. It should be noted, that these decelerations have a much shorter duration compared to the decelerations for experiments 3-18. The values of $H_{I} C_{36}$ and bending moments for experiments 
15-17 are still well below the critical limits.

Based on the results of these experiments, it can be stated that overall potential of the DLR-RMS to harm a passenger is very low. However, we plan to carry out further tests to assess the new seating arrangement as and when its installed. Secondly, since the criterion used to determine neck injuries in side impacts are still in a nascent stage [26], more tests should be undertaken to comprehensively address the situations that can arise from these loading scenarios. We also plan to focus our research efforts to address the issue of 'passenger comfortability' (i.e. prevention of motion sickness) in the DLR-RMS.

\section{ACKNOWLEDGEMENTS}

The authors would like to thank the anonymous reviewers for their valuable comments and suggestions to improve the quality of the paper. They are also grateful to Dr. Claudio Castellini for his guidance and support.

\section{REFERENCES}

[1] D. Stewart, "A platform with six degrees of freedom," in Proceedings of the Institution of Mechanical Engineers 1847-1982 (vols 1-196), vol. Volume 180 / 1965. Sage Publications, 1965, pp. 371-386.

[2] M. Bruenger-Koch, "Motion parameter tuning and evaluation for the dlr automotive simulator," December 2005.

[3] "Kuka entertainment." [Online]. Available: http://www.kukaentertainment.com/en/

[4] "4d simulator." [Online]. Available: http://www.kukaentertainment.com/en/products/robocoaster/4d_simulator/

[5] J. Heindl, M. Otter, H. Hirschmueller, M. Fromberger, F. Siegert, and H. Heinrich, "The robocoaster simulation platform, path and video generation for an authentic mars flight simulation," in Proceedings ISR-2006, Joint Conference on Robotics / ROBOTIK 2006, 2006. [Online]. Available: http://elib.dlr.de/51242/

[6] T. Bellmann, M. Otter, J. Heindl, and G. Hirzinger, "Realtime pathplanning for an interactive and industrial robot based motion simulator," in Motion Simulator Conference, 09 2007. [Online]. Available: http://elib.dlr.de/52979/

[7] T. Bellmann, "An innovative driving simulator: Robocoaster," in FISITA 2008, 2008. [Online]. Available: http://elib.dlr.de/54929/

[8] T. Bellmann, J. Heindl, M. Hellerer, R. Kuchar, K. Sharma, and G. Hirzinger, "The dlr robot motion simulator part i: Design and setup," in IEEE International Conference on Robotics and Automation (ICRA), may 2011, pp. $4694-4701$.

[9] T. Bellmann, M. Otter, and G. Hirzinger, "The dlr robot motion simulator part ii: Optimization based path-planning," in IEEE International Conference on Robotics and Automation (ICRA), may 2011, pp. 4702 -4709 .

[10] Regional court Bochum, "Decision ref. 6 o 225/95," 22.5.1996.

[11] K. Sharma, S. Haddadin, J. Heindl, T. Bellmann, S. Parusel, T. Rokahr, S. Minning, and G. Hirzinger, "Serial kinematics based motion simulator - evaluation of safety of the passenger," in The International Conference on the Safety of Industrial Automated Systems (SIAS), 2012.

[12] Multiple, "Anthropomorphic dummies for crash and escape systems testing," Advisory Group for Aerospace Research and Development (AGARD), NATO, Tech. Rep., July 1996. [Online]. Available: http://www.dtic.mil/cgibin/GetTRDoc?AD=ADA313660Location=U2doc=GetTRDoc.pdf

[13] M. Kleer, O. Hermanns, K. Dressler, and S. Mueller, "Driving simulations for commercial vehicles- a technical overview of a robot based approach," in Driving Simulation Conference 2012, September 2012.

[14] M. Voshell, "High acceleration and the human body," November 2004. [Online]. Available: http://csel.eng.ohio-state.edu/voshell/gforce.pdf

[15] S. Haddadin, A. Albu-Schäffer, and G. Hirzinger, "Safety evaluation of physical human-robot interaction via crash-testing," in Science and Systems Conference, June 2007, pp. 217-224. [Online]. Available: http://elib.dlr.de/51180/
[16] D. Munoz, A. Mansilla, F. Lopez-Valdes, and R. Martin, "A study of current neck injury criteria used for whiplash analysis. proposal of a new criterion involving upper and lower neck load cells," University of Valladolid, Tech. Rep. 05-0313, 2005.

[17] Mertz and Patrick, "Investigation of the kinematics and kinetics of whiplash," SAE Technical Paper, Tech. Rep., 1967.

[18] M. v. Horst, "Human head neck response in frontal, lateral and rear end impact loading," Ph.D. dissertation, Eindhoven University of Technology, 2002.

[19] Frontal Impact Testing Protocol, European New Car Assessment Programme (Euro NCAP) Std., Rev. 6, August 2012. [Online]. Available: http://www.euroncap.com/Content-WebPage/fb5e236e-b11b-4598-8e20-3eced15ce74e/protocols.aspx

[20] N. Yoganandan, F. Pintar, J. Zhang, B. Stemper, and M. Philippens, "Upper neck forces and moments and cranial angular accelerations in lateral impact," Annals of Biomedical Engineering, vol. 36, pp. 406-414, 2008, 10.1007/s10439-007-9422-7. [Online]. Available: http://dx.doi.org/10.1007/s10439-007-9422-7

[21] H. Henn, "Crash tests and the head injury criterion," Teaching Mathematics and its Applications, pp. 162-170, 1998.

[22] Crash Analysis Criteria Description, Workgroup Data Processing Vehicle Safety, 2006.

[23] Assessment Protocol- Adult Occupant Protection, European New Car Assessment Programme (Euro NCAP) Std., Rev. 5.4, November 2011. [Online]. Available: http://www.euroncap.com/Content-WebPage/fb5e236e-b11b-4598-8e20-3eced15ce74e/protocols.aspx

[24] O. Bostroem, M. Y. Svensson, B. Aldman, H. A. Hansson, Y. Haaland, P. Loevsund, T. Seeman, A. Suneson, A. Saeljoe, and T. Oertengren, "A new neck injury criterion candidate-based on injury findings in the cervical spinal ganglia after experimental neck extension trauma," in Proceedings Of The 1996 International Conference On The Biomechanics Of Impact (IRCOBI), Dublin, Ireland, no. 00767056, 1996, pp. 123-36.

[25] Side Impact Testing Protocol, European New Car Assessment Programme (Euro NCAP) Std., Rev. 6, August 2012. [Online]. Available: http://www.euroncap.com/Content-WebPage/fb5e236e-b11b-4598-8e20-3eced15ce74e/protocols.aspx

[26] N. Yoganandan, J. Humm, F. Pintar, C. Wolfla, and D. J. Maiman, "Lateral neck injury assessments in side impact using post mortem human subject tests," Annals of Advances in Automotive Medicine / Annual Scientific Conference, vol. 55, pp. 169-179, 2011.

[27] KUKA Robot Sensor Interface 2.2, KUKA Roboter GmbH, November 2008.

[28] KUKA System Software 5.2, 5.3, 5.4 Operating and Porgramming Instructions for System Integrators, 0th ed., KUKA Roboter $\mathrm{GmbH}$, 2007.

[29] KUKA KR C2/C3 Expert Programming, KUKA Roboter GmbH, September 2003 